\section{Model Reduction Based on Regional Pole and Covariance Equivalent Realizations}

\author{
Zidong Wang and H. Unbehauen
}

\begin{abstract}
In this paper a novel model reduction problem is studied for linear continuous-time time-invariant stochastic systems. The purpose of this problem is to design the reduced-order model so that it has the same dominant pole region and steady-state output covariance as those of the original full-order model. The resulting reduced-order model can approximate the corresponding original full-order model in two important aspects, i.e., transient and steady-state performances. Necessary and sufficient and conditions for the existence of desired reduced-order models are established, and an explicit expression for these reduced-order models is also presented. An illustrative example is used to demonstrate the effectiveness of the proposed design method.
\end{abstract}

Index Terms - Approximation theory, covariance equivalent realizations, dominant pole region, linear continuous-time stochastic systems, model reduction.

\section{INTRODUCTION}

A great many approaches are available in the literature on the general topic of model reduction, including aggregation methods [1], balancing techniques [2], Hankel norm approximation methods [3], $H_{\infty}$ norm approximations [4], and $q$-Markov covariance equivalent realizations [6], [7], [14], [15], to name just a few. A major drawback of each of these methods (with the exception of the $q$-Markov covariance equivalent realizations) is that the reduced-order models are not guaranteed to match any of the second-order information (i.e., covariance values) of the original model outputs, which is an important criterion when output performance is an item of interest.

Many engineering systems have performance requirements stated in terms of steady-state output covariance values (antenna pointing [5], vibration control in flexible structures [6], etc.). In [6], [7], [14], and [15] a projection method has been used to obtain reducedorder models that match the first $q+1$ output covariances and the first $q$-Markov parameters of the original model. These reduced models are called $q$-Markov covariance equivalent realizations, or " $q$-Markov covariance equivalent realizations (COVER's)." Due to the preservation of $q>0$ output covariances, the $q$-Markov COVER provides a reduced-order model for continuous- and discrete-time systems that has the same steady-state covariance values of each of the multiple outputs as the original model, and due to the preservation of $q$-Markov parameters the $q$-Markov COVER also has the capacity to provide good transient approximation. However, complex transformations to specific coordinates were required in the literature associated with $q$-Markov COVER's (see, for example, [14] and [15]).

Manuscript received November 13, 1996; revised May 11, 1998. Recommended by Associate Editor, H. Ozbay. The work of Z. Wang was supported by the Alexander von Humboldt Fundation of Germany and the National Natural Science Foundation of China.

Z. Wang is with the Department of Mathematics, University of Kaiserslautern, D-67663 Kaiserslautern, Germany, on leave from the Department of Automatic Control, Nanjing University of Science and Technology, Nanjing 210094, China.

H. Unbehauen is with the Automatic Control Laboratory, Faculty of Electrical Engineering, Ruhr-University Bochum, D-44780 Bochum, Germany (e-mail: unbehauen@esr.ruhr-uni-bochum.de).

Publisher Item Identifier S 0018-9286(99)07880-0.
It is well known that the transient properties of linear time-invariant systems are influenced directly by the location region of the dominant poles, and hence the regional pole assignment problem has received great attention in recent years [8]-[10]. Therefore, instead of the $q$ Markov parameters used in [6], [7], [14], and [15], we utilize the dominant pole region to represent the transient performance index. It is clear that the region in which the dominant poles are situated and the steady-state output covariance value are closely related, respectively, to the transient and steady-state performance of linear time-invariant stochastic systems. Therefore, in addition to the wellstudied covariance matching criterion, another new model reduction criterion is simultaneously proposed in this paper, i.e., the reducedorder models should preserve not only the same steady-state output covariance value but also the same dominant pole region as those of the original full-order models. This motivates the investigation of a new model reduction approach called regional pole and covariance equivalent realizations (RPCOVER's).

In the present paper we develop a novel model reduction approach for continuous-time time-invariant stochastic systems. The main contribution is twofold: 1) the conception of RPCOVER's is introduced for the reduced-order models matching both the dominant pole region and steady-state output covariance of the full-order model and 2) necessary and sufficient conditions for the existence of the expected RPCOVER's are given, and a constructive approach to designing the RPCOVER's is also presented. Specifically, the main idea proposed in this paper is to create a reduced-order model by matching the output covariance of the original model and keeping the dominant poles in the same region as those of the original model, hence, in some sense, ensuring respectively good steady-state and transient approximation between the full- and reduced-order models. The problem of regional pole and covariance equivalent realizations was investigated in [11] for discrete-time stochastic systems where the state covariance of the reduced-order model is required to equal the output covariance of the full-order model; hence this paper will focus on the continuous-time case and the output covariances of both the full- and reduced-order models are expected to be the same.

\section{Problem Formulation AND Preliminaries}

Consider a linear time-invariant continuous-time stochastic system described by

$$
\dot{x}(t)=A x(t)+D v(t), \quad y(t)=C x(t)
$$

where $x(t) \in R^{n}, v(t) \in R^{r}, y(t) \in R^{p}$, and $A, D, C$ are constant matrices with appropriate dimensions. $v(t)$ is a zero mean white noise process with covariance $\delta(t) I[\delta(t)$ is the Dirac impulse function], and $v(t)$ and $x(0)$ are uncorrelated. We assume that the matrix $A$ is Hurwitz and $(A, D)$ is controllable.

The steady-state covariance of system (1) defined by

$$
X=\lim _{t \rightarrow \infty} E\left[x(t) x^{T}(t)\right]
$$

is the unique positive definite solution to the Lyapunov equation

$$
A X+X A^{T}+D D^{T}=0 .
$$

From (1) and (2), it is easy to obtain the steady-state output covariance of system (1) as follows:

$$
\begin{aligned}
Y & =\lim _{t \rightarrow \infty} E\left[y(t) y^{T}(t)\right]=C X C^{T} \\
& =C\left[\int_{0}^{\infty} e^{A t}\left(D D^{T}\right) e^{A^{T} t} d t\right] C^{T} .
\end{aligned}
$$


Throughout this paper, we suppose that the dominant poles of system (1) are located within a circular region $D(q, r)$ in the lefthalf complex plane with the center at $q+j 0(q<0)$ and the radius $r(r<-q)$. Also, we assume that the steady-state output covariance $Y$ of (1), which can be calculated directly from (3), is known. Now, we are in a position to formulate the problem under study as follows: construct a $m$ th-order $(p \leq m<n)$ linear, continuous-time, stochastic system

$$
\dot{x}_{m}(t)=A_{m} x_{m}(t)+D_{m} w(t), \quad y_{m}(t)=C_{m} x_{m}(t)
$$

where $x_{m}(t) \in R^{m}, y_{m}(t) \in R^{p}, w(t)$ is a zero mean Gaussian white noise process with unit intensity and its order is to be determined, or equivalently, find the matrices $A_{m}, D_{m}, C_{m}$ [denoted as a triple $\left.\left(A_{m}, D_{m}, C_{m}\right)\right]$ such that the following reduction requirements are simultaneously met.

1) The poles of (4) are located inside the desired circular region $D(q, r)$.

2) The steady-state output covariance of the reduced-order model (4) is equal to that of the original full-order model (1), i.e., $Y_{m}=Y$, where

$$
\begin{aligned}
Y_{m} & =\lim _{t \rightarrow \infty} E\left[y_{m}(t) y_{m}^{T}(t)\right]=C_{m} X_{m} C_{m}^{T} \\
X_{m} & =\lim _{t \rightarrow \infty} E\left[x_{m}(t) x_{m}^{T}(t)\right]
\end{aligned}
$$

and $Y$ is defined in (3).

If the above reduction indices 1) and 2) are satisfied, then (4) is said to be a regional pole covariance equivalent realization (RPCOVER) of system (1) and the reduction task is accomplished.

\section{Main Results And Proofs}

In this section we will give the existence conditions and constructive design approach of the desired reduced-order model. To start with, we first present three important lemmas.

Lemma 1 [5], [10]: Let $M \in R^{s \times t}$ and $N \in R^{s \times z}(s \leq$ $z)$. There exists a matrix $V$ which simultaneously satisfies $N=$ $M V, V V^{T}=I$ if and only if $M M^{T}=N N^{T}$.

The following lemma is easily proved.

Lemma 2: A triple $\left(A_{m}, D_{m}, C_{m}\right)$ is a desired RPCOVER if and only if: 1) the eigenvalues of $A_{m}$ are situated within $D(q, r)$ and 2) the following algebraic equations are satisfied:

$$
\begin{gathered}
A_{m} X_{m}+X_{m} A_{m}^{T}+D_{m} D_{m}^{T}=0 \\
Y=C_{m} X_{m} C_{m}^{T} .
\end{gathered}
$$

Lemma 3 [8]: Consider the following algebraic matrix equation:

$$
-q A_{m} P-q P A_{m}^{T}+A_{m} P A_{m}^{T}+\left(q^{2}-r^{2}\right) P=-Q
$$

where $Q>0$ is arbitrary. Then the poles of $A_{m}$ are situated in the given circular region $D(q, r)$ if and only if there exists a positive definite solution $P$ to (7).

In view of the above lemmas, the main steps for designing the desired RPCOVER's can now be stated as follows. First, for a given steady-state output covariance $Y \in R^{p \times p}$, the necessary and sufficient conditions are studied for the existence of a matrix $C_{m} \in$ $R^{p \times m}$ and a positive definite matrix $X_{m} \in R^{m \times m}$ which satisfy $Y=C_{m} X_{m} C_{m}^{T}$, and then the matrices $C_{m}, X_{m}$ are determined. The obtained $X_{m}>0$ is denoted as the expected state covariance of the reduced-order model. Second, for the specified state covariance $X_{m}>0$ and circular pole region $D(q, r)$, we seek the parameters $A_{m}, D_{m}$ which simultaneously meet (5) and (7) for some $P>0$ and $Q>0$, and hence the resulting triple $\left(A_{m}, D_{m}, C_{m}\right)$ is just a desired RPCOVER.
Consider the equation $Y=C_{m} X_{m} C_{m}^{T}$ where $Y \in R^{p \times p}$ is given but is not necessarily symmetric, and $C_{m} \in R^{p \times m}, X_{m} \in R^{m \times m}$ are unknown matrices with given dimensions. We first establish the solvability of $Y=C_{m} X_{m} C_{m}^{T}$ for $C_{m} \in R^{p \times m}$ and $0<X_{m} \in$ $R^{m \times m}$. Write the singular value decomposition of $C_{m}$ as

$$
C_{m}=\left[\begin{array}{ll}
U_{c 1} & U_{c 2}
\end{array}\right]\left[\begin{array}{cc}
\Sigma_{c} & 0 \\
0 & 0
\end{array}\right]\left[\begin{array}{l}
V_{c 1}^{T} \\
V_{c 2}^{T}
\end{array}\right]
$$

where $\Sigma_{c} \in R^{r_{c} \times r_{c}}\left(r_{c}=\operatorname{rank}\left(C_{m}\right)\right)$ is the diagonal matrix consisting of all the nonzero singular values of $C_{m}, U_{c}=\left[\begin{array}{ll}U_{c 1} & U_{c 2}\end{array}\right]$ and $V_{c}=\left[\begin{array}{ll}V_{c 1} & V_{c 2}\end{array}\right]$ are orthogonal matrices with proper dimensions.

Lemma 4 [12]: There exists a solution $X_{m}$ to the equation $Y=$ $C_{m} X_{m} C_{m}^{T}$ ( $Y \in R^{p \times p}$ is not necessarily symmetric here) if and only if

$$
C_{m} C_{m}^{+} Y\left(C_{m}^{T}\right)^{+} C_{m}^{T}=Y .
$$

Corollary 1: Equation (9) is equivalent to

$$
U_{c 2}^{T} Y=0, \quad Y U_{c 2}=0 .
$$

The proof of Corollary 1 is contained in that of the following theorem.

Theorem 1: Assume that (9) or (10) holds. Then we have the following results.

1) All solutions to $Y=C_{m} X_{m} C_{m}^{T}$ are given by

$$
X_{m}=V_{c}\left[\begin{array}{cc}
\Phi & \hat{X}_{12} \\
\hat{X}_{21} & \hat{X}_{22}
\end{array}\right] V_{c}^{T}
$$

where $\Phi=\Sigma_{c}^{-1} U_{c 1}^{T} Y U_{c 1} \Sigma_{c}^{-1} \in R^{r_{c} \times r_{c}}, r_{c}=\operatorname{rank}\left(C_{m}\right)$ and $\hat{X}_{12}, \hat{X}_{21}, \hat{X}_{22}$ are arbitrary matrices with proper dimensions.

2) A positive definite solution $X_{m}$ to $Y=C_{m} X_{m} C_{m}^{T}$ exists if and only if $\Phi>0$.

Proof: See the Appendix.

Corollary 2: It is clear that for given $Y>0$ and $\Sigma_{c}>0, \Phi>0$ if and only if $U_{c 1}$ is of full row rank.

Remark 1: It follows from Theorem 1 and Corollaries 1 and 2 that to obtain the appropriate $C_{m}$ and $X_{m}>0$ which meet $Y=C_{m} X_{m} C_{m}^{T}$, one can first construct an orthogonal matrix $U_{c}=\left[\begin{array}{ll}U_{c 1} & U_{c 2}\end{array}\right]$ where $U_{c 1}$ is full row rank and $U_{c 2}$ satisfies (10). Then a diagonal positive definite matrix $\Sigma_{c} \in R^{r_{c} \times r_{c}}$ and an orthogonal matrix $V_{c}$ have to be chosen in order to obtain the parameter $C_{m}$ from (8). Subsequently, the positive definite matrix $X_{m}>0$ can be easily obtained from (11). It should be pointed out that the set of $C_{m}$ and $X_{m}>0$ which meets $Y=C_{m} X_{m} C_{m}^{T}$ is never empty. In fact, for a given positive definite matrix $Y \in R^{p \times p}$, observing that $p \leq m$, we can always set

$$
C_{m}=\left[\begin{array}{ll}
I_{p} & 0_{p \times(m-p)}
\end{array}\right], \quad X_{m}=\left[\begin{array}{cc}
Y & 0 \\
0 & I_{m-p}
\end{array}\right]
$$

and $Y=C_{m} X_{m} C_{m}^{T}$ holds naturally.

Now, our task is to study the existence conditions as well as the design approach of the desired RPCOVER's. It can be assumed from Theorem 1 that the expected state covariance $X_{m}$ for the reduced-order model is known.

Theorem 2: Given the full-order model (1), the dominant pole region $D(q, r)$, and the expected state covariance $X_{m}$ of the reducedorder system, there exists a reduced-order model (4) whose poles are located within $D(q, r)$ and state covariance equals $X_{m}$, if and only if there exist positive definite matrices $P>0$ and $Q>0$ satisfying

$$
\begin{aligned}
r^{2} P-Q & \geq 0 \\
\left(T V S^{-1}+q I\right) X_{m}+X_{m}\left(T V S^{-1}+q I\right)^{T} & \leq 0
\end{aligned}
$$


where $V$ is arbitrary orthogonal, and $S$ and $T$ are the square roots of $P$ and $r^{2} P-Q$, i.e., $S S^{T}=P, T T^{T}=r^{2} P-Q, S, T \in R^{m \times m}$. Furthermore, if (12) and (13) are met, the two parameters of the reduced-order model (4) can be obtained as follows:

$$
\begin{aligned}
& A_{m}=T V S^{-1}+q I \\
& D_{m}=\left[-\left(T V S^{-1}+q I\right) X_{m}-X_{m}\left(T V S^{-1}+q I\right)^{T}\right]^{1 / 2} .
\end{aligned}
$$

Proof-Necessity: If there exists a lower-order model (4) which satisfies the prescribed regional pole and state covariance constraints, then from Lemmas 2 and 3, there must exist $P>0$ and $Q>0$ such that

$$
-q A_{m} P-q P A_{m}^{T}+A_{m} P A_{m}^{T}+\left(q^{2}-r^{2}\right) P=-Q
$$

and

$$
A_{m} X_{m}+X_{m} A_{m}^{T}+D_{m} D_{m}^{T}=0 .
$$

Note that (14) can be rewritten as

$$
\left(A_{m}-q I\right) P\left(A_{m}-q I\right)^{T}=r^{2} P-Q
$$

and then (12) follows directly. Define $P=S S^{T}, r^{2} P-Q=T T^{T}$, then (16) can be rearranged as

$$
\left[\left(A_{m}-q I\right) S\right]\left[\left(A_{m}-q I\right) S\right]^{T}=T T^{T} .
$$

From Lemma 1, there exists an orthogonal matrix $V$ which satisfies $\left(A_{m}-q I\right) S=T V$ or

$$
A_{m}=T V S^{-1}+q I .
$$

Substituting (18) into (15) yields (13) immediately.

Sufficiency: Suppose that there exist positive definite matrices $P, Q$ and an orthogonal matrix $V$ such that (12) and (13) hold. We can directly choose $A_{m}=T V S^{-1}+q I$ and $D_{m}=\left(-\left(T V S^{-1}+\right.\right.$ $\left.q I) X_{m}-X_{m}\left(T V S^{-1}+q I\right)^{T}\right)^{1 / 2}$ where $P=S S^{T}$ and $r^{2} P-Q=$ $T T^{T}$ and then have

$$
A_{m} X_{m}+X_{m} A_{m}^{T}+D_{m} D_{m}^{T}=0 .
$$

Furthermore, from the proof of necessity, we know that $A_{m}=$ $T V S^{-1}+q I$ is equivalent to

$$
\left(A_{m}-q I\right) P\left(A_{m}-q I\right)^{T}=r^{2} P-Q .
$$

Then, it follows from Lemma 3 that $A_{m}$ satisfies the prespecified circular pole constraints. Finally, by the definition of RPCOVER, we know that the obtained triple $\left(A_{m}, D_{m}, C_{m}\right)$ is just a desired RPCOVER of the full-order model (1), where $C_{m}$ is determined by Theorem 1.

Remark 2: The positive definite matrices $P$ and $Q$ which meet (12) and (13) always exist. In fact, note that $q<0$, and therefore we can choose $P>0$ and $Q>0$ such that $T$ and $S^{-1}$ are small enough (in the norm setting) to meet (13). In the limiting case, by choosing $Q=r^{2} P$ and hence $T=0$, (13) holds automatically.

Remark 3: Note that the desired RPCOVER's are usually not unique. This freedom can be utilized to achieve new reduction requirements, such as the $H_{\infty}$-norm approximation constraint, i.e., the reduced-order models should retain the same (or similar) disturbance rejection behavior (in a $H_{\infty}$-norm sense) as the full-order model. This will be one of the main topics of further research.

Finally, the RPCOVER's based model reduction procedure can be formulated as follows.

1) Determine the output covariance $C X C^{T}$ and the dominant pole region $D(q, r)$ of the original system (1).

2) Obtain the parameter $C_{m}$ and the desired state covariance $X_{m}$ of the reduced-order model by using Theorem 1.

3) Choose $P$ and $Q$ appropriately such that (12) and (13) hold.

4) Calculate the parameters $A_{m}, D_{m}$ of the reduced-order model from Theorem 2.

\section{An Illustrative Example}

Consider the linear continuous stochastic system (1) with the parameters

$$
\begin{aligned}
A & =\left[\begin{array}{rrrrr}
-2.4983 & -0.2631 & -0.8286 & 0.8107 & 0.4423 \\
-0.3464 & -4.1546 & -1.6877 & 0.1128 & 0.5459 \\
-0.0922 & -0.2321 & -4.1253 & 0.1934 & 0.2425 \\
-0.0666 & -0.1867 & -0.0328 & -4.1349 & 0.7956 \\
-0.2554 & -0.1523 & -0.0585 & 0.1226 & -3.2082
\end{array}\right] \\
D & =\left[\begin{array}{ccccc}
0.2200 & 0 & 0 & 0 & 0 \\
0 & 0 & 0.1300 & 0 & 0 \\
0 & 0 & 0 & 0 & 0.06
\end{array}\right]^{T} \\
C & =\left[\begin{array}{lllll}
1 & 0 & 0 & 0 & 0 \\
0 & 0 & 1 & 0 & 0 \\
0 & 0 & 0 & 0 & 1
\end{array}\right] .
\end{aligned}
$$

It is easy to calculate the pole set of system matrix $A$ as

$$
\begin{aligned}
& \{-2.7887+0.0870 i,-2.7887-0.0870 i,-4.6642, \\
& \quad-4.3287,-3.5510\} .
\end{aligned}
$$

The dominant pole region can be chosen as $D(-3.5,1.5)$, i.e., $q=-3.5, r=1.5$, and the steady-state covariance and output covariance of system (1) can be, respectively, calculated as the following:

$$
\begin{aligned}
X & =\left[\begin{array}{rrrrr}
0.0097 & -0.0004 & -0.0004 & -0.0001 & -0.0004 \\
-0.0004 & 0.0002 & -0.0004 & 0.0000 & 0.0001 \\
-0.0004 & -0.0004 & 0.0021 & 0.0000 & 0.0000 \\
-0.0001 & 0.0000 & 0.0000 & 0.0000 & 0.0001 \\
-0.0004 & 0.0001 & 0.0000 & 0.0001 & 0.0006
\end{array}\right] \\
Y & =C X C^{T} \\
& =\left[\begin{array}{rrr}
0.0097 & -0.0004 & -0.0004 \\
-0.0004 & 0.0021 & 0.0000 \\
-0.0004 & 0.0000 & 0.0006
\end{array}\right]
\end{aligned}
$$

It is desired to construct a third-order RPCOVER. From Theorem 1, we can obtain the output matrix and the state covariance, respectively, as $C_{m}=I_{3}$ and thus $X_{m}=C X C^{T}$. Subject to the constraints (12) and (13), we can choose

$$
\begin{aligned}
P & =\left[\begin{array}{lll}
1.8942 & 0.1295 & 0.3386 \\
0.1295 & 1.7321 & 0.1196 \\
0.3386 & 0.1196 & 1.0168
\end{array}\right] \\
Q & =\left[\begin{array}{lll}
4.2614 & 0.2914 & 0.7618 \\
0.2914 & 3.8932 & 0.2691 \\
0.7618 & 0.2691 & 2.2799
\end{array}\right]
\end{aligned}
$$

Then, from Theorem 2, by setting $V=I_{3}$, the parameters $A_{m}, D_{m}$ can be determined as

$$
\begin{aligned}
A_{m} & =\left[\begin{array}{rrr}
-3.4826 & -0.0007 & -0.0020 \\
-0.0016 & -3.4516 & -0.0021 \\
-0.0090 & -0.0031 & -3.4094
\end{array}\right] \\
D_{m} & =\left[\begin{array}{rrr}
0.2603 & -0.0069 & -0.0077 \\
-0.0069 & 0.1197 & 0.0008 \\
-0.0077 & 0.0008 & 0.0629
\end{array}\right] .
\end{aligned}
$$

The pole set of the reduced-order systems is $\{-3.4829,-3.4090,-3.4517\}$. It is apparent that the fulland reduced-order models have the same pole region and steady-state output covariance. Furthermore, let $y=\left(\begin{array}{lll}y_{1} & y_{2} & y_{3}\end{array}\right)^{T}$, $y_{m}=\left(\begin{array}{lll}y_{m 1} & y_{m 2} & y_{m 3}\end{array}\right)^{T}$, respectively, stand for the outputs of the full- and reduced-order models which are driven by the white noise inputs. The simulation results (Figs. 1-6) show that 


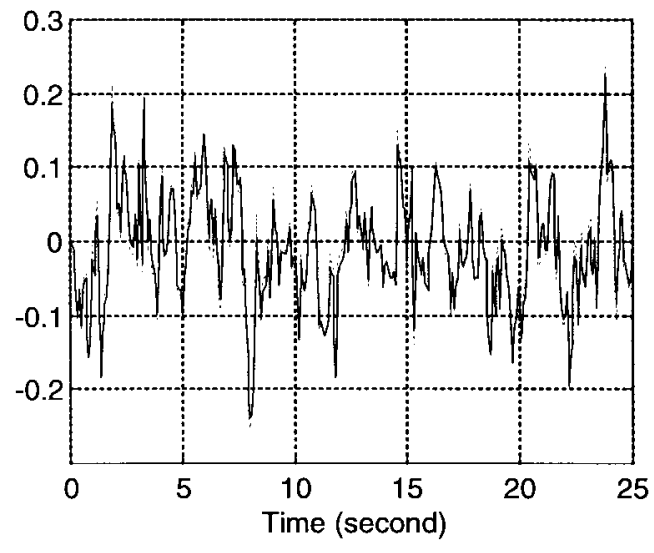

Fig. 1. "-": the output of $y_{1}$ and "...": the output of $y_{m 1}$.

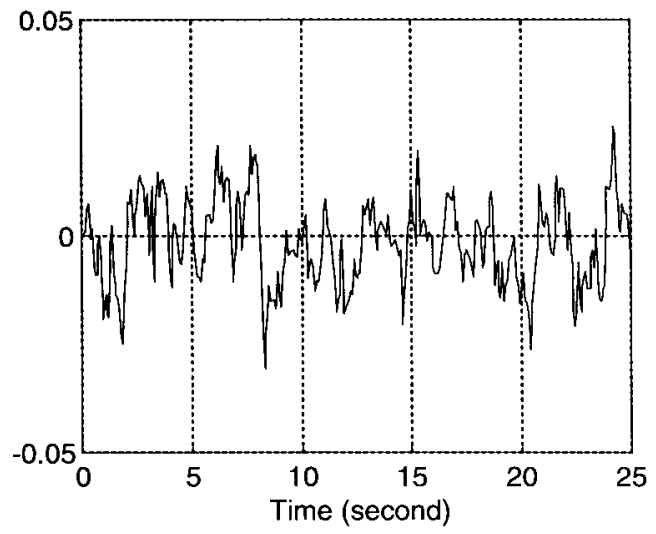

Fig. 2. The signal of $y_{1}-y_{m 1}$.

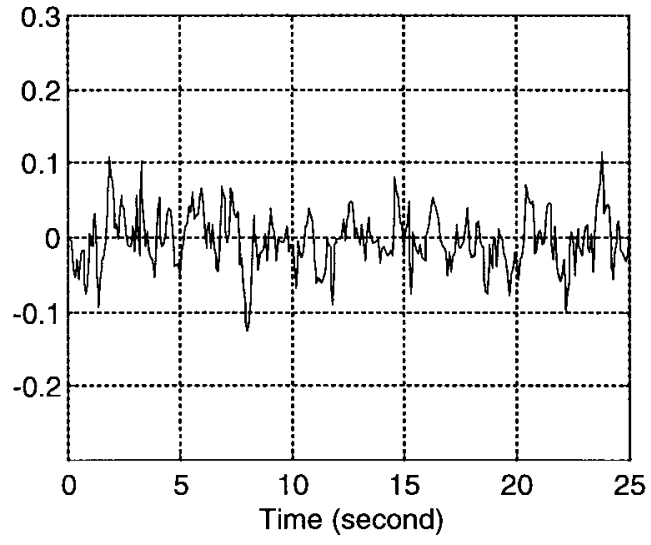

Fig. 3. "-": the output of $y_{2}$ and "...": the output of $y_{m 2}$.

the reduced-order model approximates the full-order one well and the real reduction objectives are met.

Finally, it is remarkable that, although both the RPCOVER approach developed in the present paper and the well-studied $q$ Markov COVER theory aim at designing reduced-order models which match the steady-state output covariance and approximate the transient behavior of the full-order model, in certain cases the RPCOVER approach supplements the $q$-Markov COVER theory because: 1) the dominant pole region is very often used in practice to represent the transient performance index of linear systems; 2) no complex transformations to specific coordinates are required in the

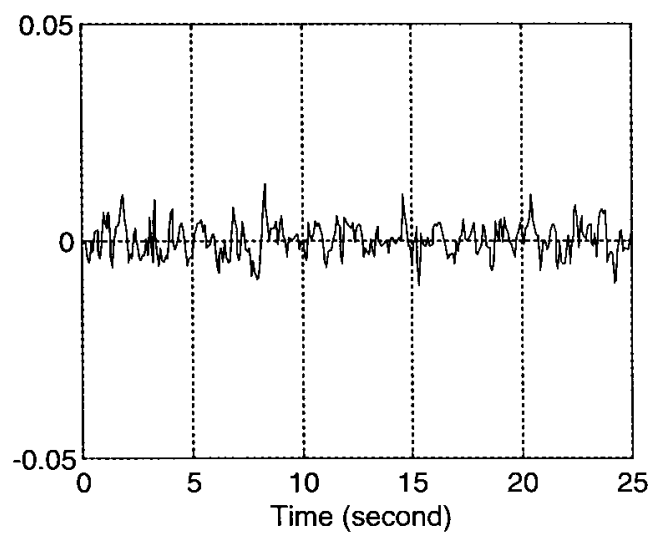

Fig. 4. The signal of $y_{2}-y_{m 2}$.

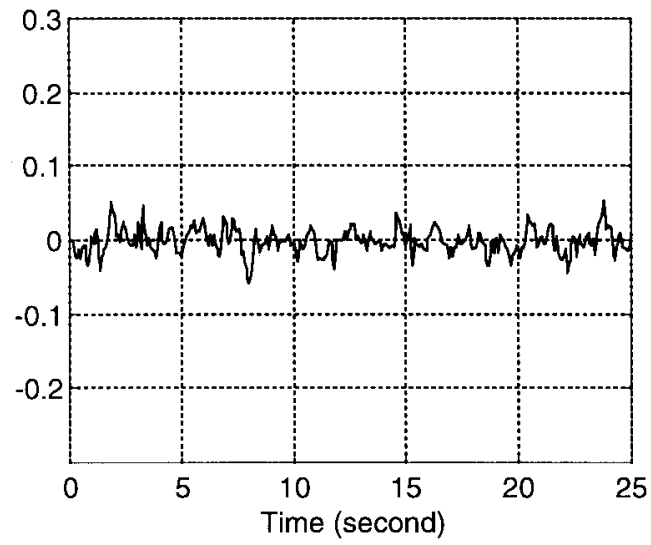

Fig. 5. "-": the output of $y_{3}$ and "...": the output of $y_{m 3}$.

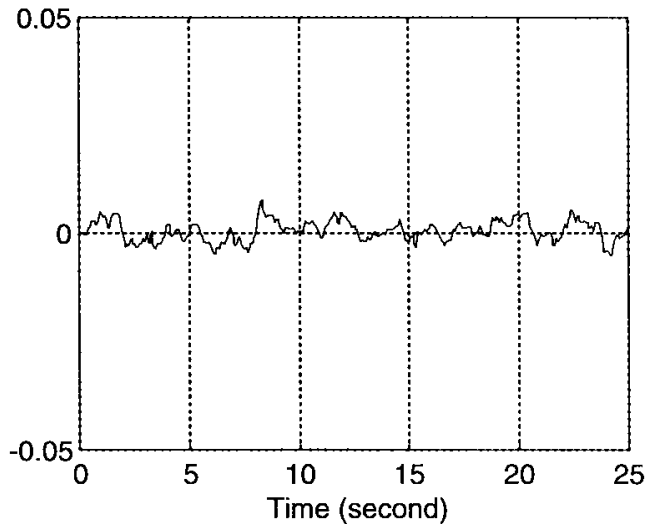

Fig. 6. The signal of $y_{3}-y_{m 3}$.

RPCOVER approach; and 3) since there exists much design freedom, the RPCOVER approach provides the possibility to realize multiple model reduction requirements.

\section{CONCLUSIONS}

This paper has developed a new approach to constructing reducedorder models which maintain both the steady-state behavior (via matching steady-state covariance) and the transient property (via matching dominant pole region). An effective algebraic method has been exploited to prove the existence of the desired reduced-order models and to derive the associated analytical expressions. Further 
studies will mainly concentrate on the extension of the present results to the case where the dominant pole region is more general (e.g., convex region) than the circular region used in this paper and on the multiple objective model reduction problem (e.g., transient and steady-state properties, disturbance rejection behavior, etc.) based on the equivalent realization of the steady-state second-order information (state covariance or output covariance).

\section{APPENDIX}

Proof of Theorem 1-1): We define

$$
\hat{X}=V_{c}^{T} X_{m} V_{c}, \quad \hat{Y}=U_{c}^{T} Y_{m} U_{c} .
$$

Then $Y=C_{m} X_{m} C_{m}^{T}$ is equivalent to

$$
\begin{aligned}
\hat{Y} & =U_{c}^{T}\left\{U_{c}\left[\begin{array}{cc}
\Sigma_{c} & 0 \\
0 & 0
\end{array}\right] V_{c}^{T} X_{m} V_{c}\left[\begin{array}{cc}
\Sigma_{c} & 0 \\
0 & 0
\end{array}\right] U_{c}^{T}\right\} U_{c} \\
& =\left[\begin{array}{cc}
\Sigma_{c} & 0 \\
0 & 0
\end{array}\right] \hat{X}\left[\begin{array}{cc}
\Sigma_{c} & 0 \\
0 & 0
\end{array}\right]
\end{aligned}
$$

which can be rewritten as

$$
\left[\begin{array}{cc}
\Sigma_{c}^{-1} & 0 \\
0 & I
\end{array}\right] \hat{Y}\left[\begin{array}{cc}
\Sigma_{c}^{-1} & 0 \\
0 & I
\end{array}\right]=\left[\begin{array}{cc}
I_{r_{c}} & 0 \\
0 & 0
\end{array}\right] \hat{X}\left[\begin{array}{cc}
I_{r_{c}} & 0 \\
0 & 0
\end{array}\right] .
$$

Partition

$$
\hat{X}=\left[\begin{array}{ll}
\hat{X}_{11} & \hat{X}_{12} \\
\hat{X}_{21} & \hat{X}_{22}
\end{array}\right], \quad \hat{Y}=\left[\begin{array}{ll}
U_{c 1}^{T} Y U_{c 1} & U_{c 1}^{T} Y U_{c 2} \\
U_{c 2}^{T} Y U_{c 1} & U_{c 2}^{T} Y U_{c 2}
\end{array}\right]
$$

then (20) is equivalent to the following equations:

$$
\begin{aligned}
\hat{X}_{11}=\Sigma_{c}^{-1} U_{c 1}^{T} Y U_{c 1} \Sigma_{c}^{-1} & =\Phi \\
\Sigma_{c}^{-1} U_{c 1}^{T} Y U_{c 2} & =0 \\
U_{c 2}^{T} Y U_{c 1} \Sigma_{c}^{-1} & =0 \\
U_{c 2}^{T} Y U_{c 2} & =0 .
\end{aligned}
$$

Noticing that a solution $X_{m}$ to $Y=C_{m} X_{m} C_{m}^{T}$ exists if and only if (23)-(25) are satisfied, and (23)-(25) are equivalent to (10), the proof of Corollary 1 follows immediately. By using the definition of $\hat{X}$, we have

$$
X_{m}=V_{c} \hat{X} V_{c}^{T}=V_{c}\left[\begin{array}{cc}
\Phi & \hat{X}_{12} \\
\hat{X}_{21} & \hat{X}_{22}
\end{array}\right] V_{c}^{T}
$$

where $\hat{X}_{12}, \hat{X}_{21}, \hat{X}_{22}$ are arbitrary matrices with proper dimensions.

2): It is clear that $X_{m}>0$ is equivalent to

$$
\left[\begin{array}{cc}
\Phi & \hat{X}_{12} \\
\hat{X}_{21} & \hat{X}_{22}
\end{array}\right]>0
$$

The necessity is obvious. To prove the sufficiency, we choose

$$
\hat{X}_{12}=\hat{X}_{21}^{T}, \quad \hat{X}_{22}=\hat{X}_{22}^{T}
$$

then the left-hand side of (26) is a partitioned Hermitian matrix. Inequality (26) holds if and only if [13]

$$
\begin{aligned}
\Phi & >0 \\
\hat{X}_{22} & >0 \\
\hat{X}_{22}-\hat{X}_{12}^{T} \Phi^{-1} \hat{X}_{12} & >0 .
\end{aligned}
$$

Since $\hat{X}_{22}$ (a symmetric matrix) and $\hat{X}_{12}\left(\hat{X}_{12}=\hat{X}_{21}^{T}\right)$ are arbitrary, for sufficiently large elements of $\hat{X}_{22}$ both (28) and (29) can be satisfied. Hence, there exists a positive definite solution $X_{m}$ to equation $Y=C_{m} X_{m} C_{m}^{T}$. The proof of Theorem 1 is then completed.

\section{ACKNOWLEDGMENT}

The authors would like to thank an anonymous referee for detailed comments and suggestions.

\section{REFERENCES}

[1] C. P. Kwong, "Optimal chained aggregation for reduced-order modeling," Int. J. Contr., vol. 35, pp. 965-982, 1982.

[2] L. Pernebo and L. M. Silverman, "Model reduction via balanced stated space representation," IEEE Trans. Automat. Contr., vol. 27, pp. 382-387, 1982.

[3] S. Kung and D. Lin, "Optimal Hankel-norm model reduction: Multivariable systems," IEEE Trans. Automat. Contr., vol. 26, pp. 832-852, 1981.

[4] W. M. Haddad and D. S. Bernstein, "Combined $L_{2} / H_{\infty}$ model reduction," Int. J. Contr., vol. 49, pp. 1523-1535, 1989.

[5] A. F. Hotz and R. E. Skelton, "Covariance control theory," Int. J. Contr., vol. 46, pp. 13-32, 1987.

[6] R. E. Skelton and E. G. Collins, Jr., "Set of $q$-Markov covariance equivalent models of discrete systems," Int. J. Contr., vol. 46, pp. 1-12, 1987.

[7] R. E. Skelton and B. D. O. Anderson, " $q$-Markov covariance equivalent realizations," Int. J. Contr., vol. 44, pp. 1477-1490, 1986.

[8] K. Furuta and S. B. Kim, "Pole assignment in a specified disk," IEEE Trans. Automat. Contr., vol. 32, pp. 423-427, 1987.

[9] W. M. Haddad and D. S. Bernstein, "Controller design with regional pole constraints," IEEE Trans. Automat. Contr., vol. 37, pp. 54-69, 1992.

[10] Z. Wang, G. Tang, and X. Chen, "Robust controller design for uncertain linear systems with circular pole constraints," Int. J. Contr., vol. 65, pp. 1045-1054, 1996.

[11] Z. Wang and Z. Guo, "An algebraic approach to regional pole and covariance equivalent realizations of discrete-time systems," Chinese J. Automation, vol. 9, pp. 311-314, 1997.

[12] A. Ben-Israel and T. N. E. Greville, Generalized Inverses: Theory and Application. New York: Wiley, 1974.

[13] E. Kreindler and A. Jameson, "Conditions for nonnegativeness of partitioned matrices," IEEE Trans. Automat. Contr., vol. 17, pp. 147-148, 1972.

[14] D. A. Wagie and R. E. Skelton, "A projection approach to covariance equivalent realization of discrete systems," IEEE Trans. Automat. Contr., vol. 31, pp. 1114-1120, 1986.

[15] A. M. King, U. B. Desai, and R. E. Skelton, "A generalized approach to $q$-Markov covariance equivalent realizations for discrete systems," Automatica, vol. 24, pp. 507-515, 1988. 